TRANSACTIONS OF THE

AMERICAN MATHEMATICAL SOCIETY

Volume 349, Number 12, December 1997, Pages 4815-4823

S 0002-9947(97)02070-9

\title{
ON MEASURES ERGODIC WITH RESPECT TO AN ANALYTIC EQUIVALENCE RELATION
}

\author{
ALAIN LOUVEAU AND GABRIEL MOKOBODZKI
}

\begin{abstract}
In this paper, we prove that the set of probability measures which are ergodic with respect to an analytic equivalence relation is an analytic set. This is obtained by approximating analytic equivalence relations by measures, and is used to give an elementary proof of an ergodic decomposition theorem of Kechris.
\end{abstract}

\section{INTRODUCTION}

In what follows, $X$ denotes a compact metrizable space, and $E$ an analytic equivalence relation on $X$, i.e. an equivalence relation on $X$ which is analytic as a subset of $X^{2}$.

Let $P(X)$ be the space of probability measures on $X$, equipped with the $w *$ topology, for which it is compact metrizable too. A measure $\mu \in P(X)$ is $E$-ergodic if for every Borel $E$-invariant set $B \subset X$, one has $\mu(B)=0$ or $\mu(B)=1$. Let $\mathcal{E}(E)$ be the set of all $E$-ergodic measures on $X$. One of the themes of this paper will be to compute the complexity of the set $\mathcal{E}(E)$ in $P(X)$. It is not hard, from its very definition, to get the upper bound $C P C A$ (or in modern notation $\Pi_{2}^{1}$ ). However, known results in particular cases indicate a much lower complexity. For example, suppose the equivalence relation $E$ is the orbit equivalence relation associated with a homeomorphism of $X$. Then $E$ is $F_{\sigma}$, and A.S. Kechris has proved that in this case $\mathcal{E}(E)$ is an $F_{\sigma \delta}$ subset of $P(X)$, using Hurewicz' ergodic theorem for nonsingular transformations. Moreover the computation is sharp, for if $E_{0}$ is the equivalence relation on $\mathcal{P}(\mathbb{N})$ given by $A E_{0} B \leftrightarrow A \triangle B$ is finite, then $\mathcal{E}\left(E_{0}\right)$ is not $G_{\delta \sigma}$.

The techniques of Kechris have been generalized by A. Ditzen in his thesis [D], where he shows that if $E$ is generated by a Borel action of a Polish locally compact group, $\mathcal{E}(E)$ is Borel.

In this paper, we will prove:

Theorem 2.1. If $E$ is analytic, $\mathcal{E}(E)$ is analytic.

This result will be obtained by using again an ergodic theorem, but this time for conservative positive $L^{1}$ contractions. We will also derive Ditzen's result easily from ours.

One of the motivations for studying the complexity of $\mathcal{E}(E)$ came from a recent result of Kechris $[\mathrm{Ke}]$, who proves, using strong set-theoretic hypotheses, a theorem

Received by the editors June 9, 1995.

1991 Mathematics Subject Classification. Primary 04A15, 28D99.

Key words and phrases. Analytic equivalence relation, ergodic measure, ergodic decomposition.

(C)1997 American Mathematical Society 
about decompositions of measures as integrals of $E$-ergodic measures, for any analytic equivalence relation $E$ (see section 3 for a precise statement of this result). In the proof, the use of strong set-theoretic hypotheses comes from a lack of knowledge of the complexity of:

(1) the set $\mathcal{E}(E)$, and

(2) a function which picks, for each non- $E$-ergodic measure $\mu$, a Borel $E$-invariant set $B$ with $0<\mu(B)<1$.

The result above answers (1) satisfactorily. For (2), we will show that such a choice function can be found and is Borel in case $E$ is $K_{\sigma}$. This will be enough for the application, using the following "inner approximation" result:

Theorem 3.1. For any analytic equivalence relation $E$ on $X$, and any $\mu \in P(X)$, there exists a $K_{\sigma}$ equivalence relation $E^{*} \subset E$ such that every Borel $E^{*}$-invariant set is $\mu$-a.e. equal to a Borel E-invariant set.

Theorem 2.1 and its consequences will be proved in section 2. The proof of Theorem 3.1, together with some more results on $K_{\sigma}$ equivalence relations and a proof of Kechris' result without additionnal hypotheses, will be given in section 3 . Before that, we will prove in section 1 another "approximation result" for analytic equivalence relations, which is the main tool for the other proofs.

If $\sigma$ is a probability measure on $X^{2}$, let $p_{0}(\sigma)$ and $p_{1}(\sigma)$ be its two projections on $X$. We say that a Borel set $B \subset X$ is $\sigma$-invariant if $\sigma\left(B^{2} \cup(X-B)^{2}\right)=1$.

Theorem 1.1. Let $E$ be analytic on $X$, and $\mu \in P(X)$. There exists $\sigma \in P\left(X^{2}\right)$ with the following properties:

(i) $\sigma$ is symmetric, $p_{o}(\sigma)=p_{1}(\sigma)$ is measure-equivalent to $\mu$, and $\sigma(E)=1$.

(ii) Every Borel $\sigma$-invariant set is $\mu$-a.e. equal to a Borel E-invariant set.

\section{Positive $L^{1}$ contractions and the approximation OF ANALYTIC EQUIVALENCE RELATIONS BY MEASURES}

Let us first recall some facts from the ergodic theory of positive $L^{1}$ contractions (the reader is referred for these results to Krengel's book $[\mathrm{Kr}]$ ).

Let $\sigma$ be a probability measure on $X^{2}$, and $\nu=p_{0}(\sigma)$ its first projection on $X$. We say that $\sigma$ is null-preserving if its second projection $p_{1}(\sigma)$ is absolutely continuous with respect to $\nu$ (in notation, $p_{1}(\sigma) \ll \nu$ ). One can then associate to $\sigma$ an operator $T_{\sigma}: L^{1}(\nu) \rightarrow L^{1}(\nu)$ as follows: Given $f \in L^{1}(\nu)$, the measure $f . \sigma$ is absolutely continuous with respect to $\sigma$, hence $p_{1}(f . \sigma) \ll p_{1}(\sigma) \ll \nu$, and there is a function $T_{\sigma} f \in L^{1}(\nu)$ such that $p_{1}(f . \sigma)=T_{\sigma} f . \nu$.

Clearly, the operator $T_{\sigma}$ is positive and linear, and a contraction of $L^{1}(\nu)$. Moreover, if $\left(\sigma_{x}\right)_{x \in X}$ is a disintegration of $\sigma$ with respect to $\nu$, i.e. the map $x \mapsto \sigma_{x}$ is Borel from $X$ to $P(X)$ and $\sigma=\int \sigma_{x} d \nu$, then the adjoint operator $T_{\sigma}^{*}: L^{\infty}(\nu) \rightarrow L^{\infty}(\nu)$ is given, for bounded $f$, by

$$
T_{\sigma}^{*} f(x)=\int f(y) d \sigma_{x}(y) .
$$

In particular $T_{\sigma}^{*} 1=1$, and $T_{\sigma}$ is called Markovian. (Conversely, by a result of Neveu, see $[\mathrm{Kr}]$, any Markovian positive $L^{1}(\nu)$ contraction arises in this way from some $\sigma$ with $p_{0}(\sigma)=\nu$.)

Let us assume now that $\sigma$ is symmetric, i.e. $\int f(x, y) d \sigma(x, y)=\int f(y, x) d \sigma(x, y)$ for all continuous $f$ on $X^{2}$. Then $\nu=p_{0}(\sigma)=p_{1}(\sigma)$, so $\sigma$ is null-preserving, $T_{\sigma} 1=1$, and $T_{\sigma}=T_{\sigma}^{*}$ on $L^{\infty}(\nu)$. 
Let $\mathcal{B}(\sigma)=\left\{B \subset X: B\right.$ is Borel, $\left.T_{\sigma}\left(1_{B}\right)=1_{B}\right\}$ be the $\sigma$-algebra of Borel invariant sets for $T_{\sigma}$. Then a Borel set $B$ is in $\mathcal{B}(\sigma)$ if and only if $\sigma\left(B^{2} \cup(X-B)^{2}\right)=$ 1. To see this, suppose first $B \in \mathcal{B}(\sigma)$. Then by definition of $T_{\sigma}, p_{1}\left(\left.\sigma\right|_{B \times X}\right)=\left.\nu\right|_{B}$; hence $\sigma(B \times(X-B))=0$. By symmetry, $\sigma\left(B^{2} \cup(X-B)^{2}\right)=1$. Conversely, if $\sigma$ is supported by $B^{2} \cup(X-B)^{2}$, then $1_{B \times X} \cdot \sigma=1_{B^{2}} \cdot \sigma=1_{X \times B} \cdot \sigma$, so $p_{1}\left(1_{B \times X} \cdot \sigma\right)=$ $1_{B} \cdot \sigma$ and $T_{\sigma}\left(1_{B}\right)=1_{B}$, as desired. The main result that we will need is the following consequence of an ergodic theorem of Hopf (see Krengel [Kr], theorem 3.3.5) which implies that for such a symmetric $\sigma$, one has:

For any function $f \in L^{1}(\nu)$, the Cesaro averages $\frac{1}{N} \sum_{n=0}^{N-1} T_{\sigma}^{n} f$ converge pointwise $\nu$-a.e. to the conditional expectation of $f$ with respect to $\mathcal{B}(\sigma)$.

Now let $E$ be an analytic equivalence relation on $X$, and $\mu \in P(X)$. We say that $\sigma \in P\left(X^{2}\right)$ is adapted to the pair $(E, \mu)$ if $\sigma$ is symmetric, $\sigma(E)=1$, and $\nu=p_{0}(\sigma)=p_{1}(\sigma)$ is measure-equivalent to $\mu$ (i.e. $\nu \ll \mu$ and $\mu \ll \nu$ ). We denote by $\mathcal{B}(E)$ the $\sigma$-algebra of Borel $E$-invariant sets, and by $\mathcal{B}_{\mu}(E)$ and $\mathcal{B}_{\mu}(\sigma)$ respectively the images of $\mathcal{B}(E)$ and $\mathcal{B}(\sigma)$ in $L^{1}(\mu)$.

The main tool of this paper is the following:

Theorem 1.1. If $E$ is an analytic equivalence relation on $X$, and $\mu$ is a probability measure on $X$, there exists $\sigma \in P\left(X^{2}\right)$ which is adapted to $(E, \mu)$ and satisfies $\mathcal{B}_{\mu}(E)=\mathcal{B}_{\mu}(\sigma)$.

Proof. First note that if $B$ is Borel and $E$-invariant, then $E \subset B^{2} \cup(X-B)^{2}$; hence $B \in \mathcal{B}(\sigma)$ for all adapted measures $\sigma$. This shows that $\mathcal{B}_{\mu}(E) \subset \mathcal{B}_{\mu}(\sigma)$ for all adapted $\sigma$.

To prove the result, we first claim it is enough to prove

$$
\mathcal{B}_{\mu}(E)=\bigcap\left\{\mathcal{B}_{\mu}(\sigma): \sigma \text { adapted to }(E, \mu)\right\}
$$

To see this, note that each $\mathcal{B}_{\mu}(\sigma)$ is a $\sigma$-algebra in $L^{1}(\mu)$, hence is norm-closed. So, as $L^{1}(\mu)$ is separable, one gets from $(*)$ the existence of a sequence $\left(\sigma_{n}\right)_{n>0}$ of adapted measures with $\mathcal{B}_{\mu}(E)=\bigcap_{n} \mathcal{B}_{\mu}\left(\sigma_{n}\right)$. Now it is easy to check that if $\sigma_{1}$ and $\sigma_{2}$ are adapted with $\sigma_{1} \ll \sigma_{2}$, then $\mathcal{B}_{\mu}\left(\sigma_{2}\right) \subset \mathcal{B}_{\mu}\left(\sigma_{1}\right)$. So, if we set $\sigma=$ $\sum_{n>0} 2^{-n} . \sigma_{n}$, we get an adapted measure $\sigma$ which satisfies $\mathcal{B}_{\mu}(E)=\mathcal{B}_{\mu}(\sigma)$.

It remains to prove the inclusion from right to left in $(*)$. So fix a Borel set $B \subset X$ such that its class in $L^{1}(\mu)$ is in $\bigcap\left\{\mathcal{B}_{\mu}(\sigma): \sigma\right.$ adapted to $\left.(E, \mu)\right\}$. This means that for any $\sigma$ adapted to $(E, \mu)$, there is $B^{*}$ Borel with $\mu\left(B \triangle B^{*}\right)=0$ such that $\sigma$ is supported by $B^{* 2} \cup\left(X-B^{*}\right)^{2}$. But as $\mu \ll p_{0}(\sigma)$, we get that $\sigma$ is also supported by $B^{2} \cup(X-B)^{2}$.

Our goal is to find a Borel set $B^{*}$ which is $E$-invariant and such that $\mu\left(B \triangle B^{*}\right)=$ 0 .

Set $c(B)=\inf \left\{\mu\left(B^{\prime}\right): B^{\prime}\right.$ is $E$-invariant, $\left.\mu\left(B-B^{\prime}\right)=0\right\}$. Clearly $\mu(B) \leq c(B)$, and as the infimum in the right hand side is attained, it is enough to prove that $c(B) \leq \mu(B)$. To prove this inequality, we will compute $c(B)$ in a different way.

First note that if $A$ is an analytic $E$-invariant set, there is a Borel $E$-invariant set $A^{\prime}$ with $A \subset A^{\prime}$ and $\mu\left(A^{\prime}-A\right)=0$. To see this, start with some Borel $B_{0}$ containing $A$ with $\mu\left(B_{0}-A\right)=0$. Let $C_{0}=\left\{x \in X: \forall y E x y \in B_{0}\right\}$. $C_{0}$ is coanalytic, and $A \subset C_{0} \subset B_{0}$. By separation, pick $B_{1}$ Borel with $A \subset B_{1} \subset C_{0}$, and set $C_{1}=\left\{x \in X: \forall y E x y \in B_{1}\right\}$, and so on. Continuing this way, one gets decreasing sequences of Borel sets $B_{n}$ and of coanalytic $E$-invariant sets $C_{n}$ with $A \subset B_{n+1} \subset C_{n} \subset B_{n}$. Then $A^{\prime}=\bigcap_{n} B_{n}=\bigcap_{n} C_{n}$ is both Borel and $E$-invariant, and works. 
Set $[C]_{E}=\{x \in X: \exists y \in C y E x\}$. If $C$ is Borel, then $[C]_{E}$ is analytic and $E$-invariant. By the previous fact, we get

$$
1-c(B)=\sup \left\{\mu(C): C \text { is Borel and } \mu\left(B \cap[C]_{E}\right)=0\right\} .
$$

Consider then, for Borel $C, \gamma(C)=\mu\left(B \cap[C]_{E}\right)$. Let $S P(X)$ be the space of subprobability measures on $X$, i.e. of positive Radon measures $\mu$ on $X$ with $\mu(X) \leq 1$, with the $w^{*}$-topology. In $S P\left(X^{2}\right)$ define

$$
H=\left\{\theta: \theta\left(X^{2}-E\right)=0 \text { and } p_{0}(\theta) \leq\left.\mu\right|_{B}\right\} .
$$

As $E$ is analytic and $B$ is Borel, $H$ is analytic in $S P\left(X^{2}\right)$, and is measure-convex. So its image $p_{1}(H)$ under $p_{1}$ is also analytic and measure-convex in $S P(X)$. We claim that for all Borel $C$

$$
\gamma(C)=\sup _{\nu \in p_{1}(H)}(\nu(C)) .
$$

To see this, first let $\nu=p_{1}(\theta)$, with $\theta \in H$. Then

$$
\nu(C)=\theta(X \times C)=\theta\left([C]_{E} \times C\right)
$$

as $\theta$ is supported by $E$; and so $\nu(C) \leq p_{0}(\theta)\left([C]_{E}\right) \leq \mu\left(B \cap[C]_{E}\right)=\gamma(C)$. Conversely, if $A=B \cap[C]_{E}$, then for each $x \in A$ there is $y \in C$ with $y E x$, and so by the Jankov-Von Neumann selection theorem we can find a $\mu$-measurable function $\phi: A \rightarrow C$ such that $(x, \phi(x)) \in E$ for all $x \in A$. Let $\theta$ be the image of $\left.\mu\right|_{A}$ under the map $x \mapsto(x, \phi(x))$. Clearly $\theta$ is in $H$, and $p_{1}(\theta)(C)=p_{1}(\theta)(X)=p_{0}(\theta)(X)=$ $\mu(A)=\gamma(C)$, as desired.

We now use the following result: Given a measure $\mu$ on $X$, and an analytic measure-convex set $L$ in $S P(X)$, there is a unique decomposition of $\mu$ as $\mu=\mu_{0}+\mu_{1}$, with $\mu_{0}=\left.\mu\right|_{C_{0}}$ for some Borel set $C_{0}$ which satisfies $\nu\left(C_{0}\right)=0$ for all $\nu \in L$, and $\mu_{1}=\left.\mu\right|_{X-C_{0}}$ is absolutely continuous with respect to some $\nu$ in $L$.

To prove this fact, let $C_{0}$ be an essential supremum w.r.t. $\mu$ of all Borel sets $C$ with $\sup _{\nu \in L} \nu(C)=0$. Then $\mu_{0}=\left.\mu\right|_{C_{0}}$ has the desired properties. Moreover $\mu_{1}=\mu-\mu_{0}$ is absolutely continuous with respect to $\gamma_{L}=\sup \{\nu: \nu \in L\}$. Now let $C_{1}$ be an essential supremum w.r.t. $\mu$ of all Borel sets $C$ such that for some $\nu \in L$, $\left.\mu\right|_{C} \ll \nu$. Clearly, one has $\mu\left(C_{0} \cap C_{1}\right)=0$. By maximality of $C_{1}$, one also has that $\left.\mu\right|_{X-C_{1}}$ is orthogonal to all measures $\nu \in L$. One can then apply a theorem of Mokobodzki (see e.g. [KL], chapter 9): The measure $\left.\mu\right|_{X-C_{1}}$ is supported by a Borel set $D$ with $\gamma_{L}(D)=0$. So $\left.\mu\right|_{X-C_{1}}$ is supported by $C_{0}$, and $C_{1}=X-C_{0}$ $\mu$-a.e. Finally if $\left(D_{n}\right)$ are Borel sets and $\left(\nu_{n}\right)$ are measures in $L$ such that $C_{1}=$ $\bigcup_{n} D_{n}$ and $\left.\mu\right|_{D_{n}} \ll \nu_{n}$, then $\nu=\sum_{n} 2^{-n} . \nu_{n}$ is a measure in $L$ by measureconvexity, and $\left.\mu\right|_{C_{1}}=\mu_{1} \ll \nu$, as desired.

We apply this result to our computation of $c(B)$. Recall that

$$
1-c(B)=\sup \{\mu(C): C \text { Borel, } \gamma(C)=0\},
$$

where

$$
\gamma(C)=\mu\left(B \cap[C]_{E}\right)=\sup _{\nu \in p_{1}(H)}(\nu(C)) .
$$

We apply the result to $\mu$ and the analytic measure convex set $p_{1}(H)$, and get the corresponding decomposition $\mu=\mu_{0}+\mu_{1}$. By definition of $c(B)$, we have $1-c(B)=\mu_{0}(X)$, so that $c(B)=\mu_{1}(X)$. Let $\theta$ be a measure in $H$ with $\mu_{1} \ll p_{1}(\theta)$, let $f$ be such that $\mu_{1}=f \cdot p_{1}(\theta)$, and define $\theta^{\prime}$ by $d \theta^{\prime}(x, y)=f(y) \cdot d \theta(x, y)$. Clearly $\theta^{\prime}$ is supported by $E$, as $\theta$ is. Also $p_{1}\left(\theta^{\prime}\right)=\mu_{1} \leq \mu$, and $p_{0}\left(\theta^{\prime}\right) \ll p_{0}(\theta) \leq$ 
$\left.\mu\right|_{B}$. So both $p_{0}\left(\theta^{\prime}\right)$ and $p_{1}\left(\theta^{\prime}\right)$ are absolutely continuous w.r.t. $\mu$. Let $\theta^{\prime \prime}$ be symmetric to $\theta^{\prime}$, and $\mu^{\prime}$ the image of $\mu$ under the map $x \mapsto(x, x)$. Finally set $\sigma=\frac{1}{2} \mu^{\prime}+\frac{1}{4 \theta^{\prime}(X)}\left(\theta^{\prime}+\theta^{\prime \prime}\right)$. Clearly $\sigma$ is a symmetric probability measure on $X^{2}$, and $\sigma$ is supported by $E$. Moreover, $\nu=p_{0}(\sigma)=p_{1}(\sigma)=\frac{1}{2} \mu+\frac{1}{4 \theta^{\prime}(X)}\left(p_{0}\left(\theta^{\prime}\right)+p_{1}\left(\theta^{\prime}\right)\right)$ is measure-equivalent to $\mu$, so $\sigma$ is adapted to $(E, \mu)$. By our hypothesis on $B, \sigma$ is supported by $B^{2} \cup(X-B)^{2}$; hence so is $\theta^{\prime}$. But $\left.p_{0}\left(\theta^{\prime}\right) \ll \mu\right|_{B}$, so $\theta^{\prime}$ is supported by $B^{2}$. So $c(B)=p_{1}\left(\theta^{\prime}\right)(X)=p_{1}\left(\theta^{\prime}\right)(B) \leq \mu(B)$, as desired. This finishes the proof of the theorem.

\section{The COMPlexity of $\mathcal{E}(E)$}

Theorem 2.1. If $E$ is an analytic equivalence relation on $X$, the set $\mathcal{E}(E)$ of $E$ ergodic probability measures is analytic in $P(X)$.

Proof. One has the equivalence

$\mu$ is $E$-ergodic $\leftrightarrow \exists \sigma[\sigma$ is adapted to $(E, \mu)$ and $\forall B \in \mathcal{B}(\sigma) \mu(B)=0$ or 1$]$.

The implication from right to left follows from the fact that for any adapted $\sigma$ one has $\mathcal{B}_{\mu}(E) \subset \mathcal{B}_{\mu}(\sigma)$, and the implication from left to right is Theorem 1.1.

Now note that for any adapted $\sigma, p_{0}(\sigma)$ is equivalent to $\mu$; hence in the equivalence above, the condition $\mu(B)=0$ or 1 can be replaced by $p_{0}(\sigma)(B)=0$ or 1 . Let $\left(f_{m}\right)_{m \in \mathbb{N}}$ be a sequence of continuous functions, norm-dense in $\mathcal{C}(X)$. Applying the Hopf ergodic theorem to $T_{\sigma}$ and $\nu=p_{0}(\sigma)$, we get:

$\mu$ is $E$-ergodic $\leftrightarrow \exists \sigma[(1) \sigma$ is adapted to $(E, \mu)$ and

$$
\text { (2) } \left.\forall m\left(\lim _{N \rightarrow \infty} \frac{1}{N} \sum_{n=0}^{N-1} T_{\sigma}^{n} f_{m}(x)=\int f_{m} d p_{0}(\sigma) p_{0}(\sigma) \text {-a.e. }\right)\right] \text {. }
$$

Now the set of symmetric measures is closed in $P\left(X^{2}\right)$, the relation " $p_{0}(\sigma)$ is equivalent to $\mu$ " is Borel in $P\left(X^{2}\right) \times P(X)$, and as $E$ is analytic, the set of measures supported by $E$ is analytic too. So $\{(\sigma, \mu): \sigma$ is adapted to $(E, \mu)\}$ is analytic in $P\left(X^{2}\right) \times P(X)$.

Moreover the proof of the disintegration theorem is uniform, and there exists a Borel set $C \subset P\left(X^{2}\right) \times X$ and a Borel function $\phi: C \rightarrow P(X)$ such that:

(i) $\forall \sigma \in P\left(X^{2}\right) C_{\sigma}=\{x:(\sigma, x) \in C\}$ has $p_{0}(\sigma)$-measure 1 , and

(ii) if for $(\sigma, x) \in C$ one sets $\sigma_{x}=\phi(\sigma, x)$, then $\sigma=\int_{C_{\sigma}} \sigma_{x} d p_{o}(\sigma)(x)$.

It follows that for symmetric $\sigma$ and a fixed function $f \in \mathcal{C}(X)$, there is a Borel set $D$ and Borel functions $F_{n}: D \rightarrow \mathbb{R}$ such that

(i) $D_{\sigma}=\{x:(\sigma, x) \in D\}$ has $p_{0}(\sigma)$-measure 1 , and

(ii) $\forall(\sigma, x) \in D \forall n F_{n}(\sigma, x)=T_{\sigma}^{n} f(x)$.

It then follows easily that the set of symmetric $\sigma$ 's satisfying (2) is Borel in $P\left(X^{2}\right)$, and finally the equivalence above gives an analytic definition for $\mathcal{E}(E)$, as desired.

Remarks. 1. The same result holds if $X$ is only assumed to be analytic in some Polish space. For one can pick any Polish compactification $\tilde{X}$ of $X$, and extend $E$ by equality outside $X$. The resulting equivalence relation $\tilde{E}$ on $\tilde{X}$ is still analytic, and

$$
\mu \text { is } E \text {-ergodic } \leftrightarrow \mu \text { is } \tilde{E} \text {-ergodic and } \mu(X)=1 ;
$$

hence $\mathcal{E}(E)$ is still analytic. 
2. In terms of complexity, the previous result is best possible. To see this, consider the continuous map $\phi: X^{2} \rightarrow P(X): \phi(x, y)=\frac{1}{2}\left(\delta_{x}+\delta_{y}\right)$. Clearly $E=\phi^{-1}(\mathcal{E}(E))$; hence $\mathcal{E}(E)$ cannot be Borel if $E$ is not Borel.

However in some situations, one is interested not in all $E$-ergodic measures, but in the subset $\mathcal{E}_{*}(E)$ consisting of the non- $E$-atomic ones, i.e. those which are not supported by an $E$-equivalence class. Being $E$-atomic is easily seen to be analytic, and non-Borel in general by the previous fact. Hence $\mathcal{E}_{*}(E)$ is the difference of two analytic sets, but its exact complexity is not known.

Corollary 2.2. Suppose that either (i) $E$ is $K_{\sigma}$ in $X^{2}$, or (ii) (Ditzen, [D]) $E$ is the orbit relation induced by some Borel action of a Polish locally compact group $G$ on $X$, i.e. for some Borel action $\alpha: G \times X \rightarrow X$, one has $x E y \leftrightarrow \exists g \in G \alpha(g, x)=y$. Then $\mathcal{E}(E)$ is Borel in $P(X)$.

Proof. By Theorem 2.1, $\mathcal{E}(E)$ is analytic in $P(X)$. So we have to prove that in both cases it is coanalytic too. Let $\mathcal{K}(X)$ be the space of compact subsets of $X$, with the Hausdorff topology, for which it is compact metrizable. One has

$$
\mu \in \mathcal{E}(E) \leftrightarrow \forall K \in \mathcal{K}(X)\left(\mu\left([K]_{E}\right)=0 \text { or } \mu\left([K]_{E}\right)=1\right)
$$

To prove this from left to right, use the remark that any analytic $E$-invariant set is $\mu$-a.e. equal to a Borel $E$-invariant set. For the other direction, note that if some Borel $E$-invariant set $B$ has $\mu$-measure different from 0 and 1 , it contains a compact set $K$ of positive $\mu$-measure, and $[K]_{E}$ also has $\mu$-measure different from 0 and 1.

For an arbitrary analytic equivalence relation $E$, the relation $x \in[K]_{E}$ is analytic, and the equivalence above gives only that $\mathcal{E}(E)$ is $C P C A$, a bad upper bound in view of Theorem 2.1. But if this relation happens to be Borel, then so are the relations, in $P(X) \times \mathcal{K}(X), \mu\left([K]_{E}\right)=0$ and $\mu\left([K]_{E}\right)=1$, and the equivalence above gives that $\mathcal{E}(E)$ is coanalytic, as desired.

In case (i), the relation $x \in[K]_{E}$ is $K_{\sigma}$ in $X \times \mathcal{K}(X)$, and we are done. In case (ii), we can use a theorem of Varadarajan: There is a finer Polish topology on $X$ which makes the action $\alpha$ continuous. For this new topology, $X$ is Polish, the new $\mathcal{K}(X)$ is Polish too, and both $E$ and the relation $x \in[K]_{E}$ become Borel, as projections of Borel relations with $K_{\sigma}$ sections. The above discussion now lets us conclude the proof.

Remark. One cannot hope for a better result in case (ii), for there are equivalence relations induced by Borel actions of Polish locally compact groups which are of arbitrarily high Borel class, and the same trick as in the remark following Theorem 2.1 shows that $\mathcal{E}(E)$ is of arbitrarily high Borel class too. For $K_{\sigma} E$ 's, we will come back to this question at the end of section 3 .

\section{Approximation of analytic equivalence Relations by $K_{\sigma}$ Ones, AND KECHRIS' ERGODIC DECOMPOSITION THEOREM}

Theorem 3.1. Let $E$ be an analytic equivalence relation on $X$, and $\mu \in P(X)$. There exists a $K_{\sigma}$ equivalence relation $E^{\prime}$ on $X$ such that (i) $E^{\prime} \subset E$ and (ii) every $E^{\prime}$-invariant Borel set is $\mu$-a.e. equal to an E-invariant Borel set.

Proof. This is a simple consequence of Theorem 1.1: Pick $\sigma$ adapted to $(E, \mu)$ with $\mathcal{B}_{\mu}(\sigma)=\mathcal{B}_{\mu}(E)$. Let $K \subset X^{2}$ be a $K_{\sigma}$ subset of $E$ with $\sigma(K)=1$, and $E^{\prime}$ the equivalence relation generated by $K$. Then $E^{\prime}$ is $K_{\sigma}, E^{\prime} \subset E$, and $\sigma\left(E^{\prime}\right)=1$, so 
$\sigma$ is also adapted to $\left(E^{\prime}, \mu\right)$. And we have $\mathcal{B}_{\mu}(E) \subset \mathcal{B}_{\mu}\left(E^{\prime}\right) \subset \mathcal{B}_{\mu}(\sigma) \subset \mathcal{B}_{\mu}(E)$, as desired.

As a consequence of this result and the computations in section 2, we can now give an elementary proof of the following result of A.S. Kechris [Ke] (which he proved using extra set-theoretical assumptions)

Theorem 3.2 (Kechris). Let $E$ be an analytic equivalence relation on $X$, and $\mu \in$ $P(X)$. There exists a Borel function $\varphi: X \rightarrow P(X)$ satisfying

(i) for $\mu$-a.e. $x \in X, \mu_{x}=\varphi(x)$ is E-ergodic, $\varphi^{-1}(\varphi(x))=\left\{y \in X: \mu_{y}=\mu_{x}\right\}$ is E-invariant, and $\mu_{x}\left(\varphi^{-1}(\varphi(x))\right)=1$.

(ii) $\mu=\int \mu_{x} d \mu(x)$.

Proof. We use disintegration with respect to the algebra $\mathcal{B}_{\mu}(E)$, which is normseparable in $L^{1}(\mu)$. Fix $\left(B_{n}\right)_{n \in \mathbb{N}}$, a sequence in $\mathcal{B}(E)$ norm-dense in $\mathcal{B}_{\mu}(E)$, and define $\varphi_{0}: X \rightarrow\{0,1\}^{\mathbb{N}}$ by $\varphi_{0}(x)(n)=1_{B_{n}}(x)$. Set $\nu=\varphi_{0}(\mu)$. The measure $\nu$ is supported by the analytic set $Y=\varphi_{0}[X]$, and by the disintegration theorem, there exists a Borel map $\varphi_{1}: Y \rightarrow P(X)$ with $\mu=\int \varphi_{1}(y) d \nu(y)$, and for $\nu$-a.a. $y, \varphi_{1}(y)$ is supported by $\varphi_{0}^{-1}(y)$.

So if we set $\varphi(x)=\varphi_{1}\left(\varphi_{0}(x)\right)$, we get a Borel function which is $E$-invariant as $\varphi_{0}$ is, satisfies $\mu$-a.e. that $\varphi(x)$ is supported by $\varphi^{-1}(\varphi(x))$, and $\mu=\int \varphi(x) d \mu(x)$. So it only remains to show that $\mu$-a.e. $\varphi(x)$ is $E$-ergodic.

By 3.1, let $E^{\prime} \subset E$ be a $K_{\sigma}$ equivalence relation with $\mathcal{B}_{\mu}\left(E^{\prime}\right)=\mathcal{B}_{\mu}(E)$, so that the sequence $\left(B_{n}\right)$ is still dense in $\mathcal{B}_{\mu}\left(E^{\prime}\right)$. We claim that $\mu$-a.e. $\varphi(x)$ is $E^{\prime}$-ergodic (hence a fortiori $E$-ergodic), or equivalently that the set $A=\left\{y \in Y: \varphi_{1}(y)\right.$ is not $E^{\prime}$-ergodic $\}$ has $\nu$-measure 0 .

Assume not, for a contradiction. By 2.2, $A$ is Borel in $Y$, hence $\nu$-measurable, and $\nu(A)>0$. For each $y \in A$ there are $\epsilon>0$ and $K \in \mathcal{K}(X)$ with $\epsilon \leq \varphi_{1}(y)\left([K]_{E^{\prime}}\right) \leq$ $1-\epsilon$. By reducing $A$ to some Borel subset $A^{\prime}$ with $\nu\left(A^{\prime}\right)=a>0$, we may assume that the same $\epsilon$ works for all $y \in A^{\prime}$. Now the relation $\epsilon \leq \varphi_{1}(y)\left([K]_{E^{\prime}}\right) \leq 1-\epsilon$ is Borel in $Y \times \mathcal{K}(X)$; hence by the Jankov-von Neumann selection theorem, there is a Borel set $A^{\prime \prime} \subset A^{\prime}$ with $\nu\left(A^{\prime \prime}\right)=a$, and a Borel function: $y \in A^{\prime \prime} \mapsto K_{y} \in \mathcal{K}(X)$ such that for $y \in A^{\prime \prime} \epsilon \leq \varphi_{1}(y)\left(\left[K_{y}\right]_{E^{\prime}}\right) \leq 1-\epsilon$. Set $B=\bigcup_{y \in A^{\prime \prime}}\left(\left[K_{y}\right]_{E^{\prime}} \cap \varphi_{0}^{-1}(y)\right)$. The set $B$ is both Borel and $E^{\prime}$-invariant. And an immediate computation gives, for every $n, \mu\left(B \triangle B_{n}\right) \geq a \epsilon$, contradicting the density of the $B_{n}$ 's, and finishing the proof.

In the previous proof, we used the Jankov-von Neuman selection theorem to find, in a universally measurable way, for $E K_{\sigma}$ and $\mu \notin \mathcal{E}(E)$ some compact $K$ with $\mu\left([K]_{E}\right) \neq 0$ and $\mu\left([K]_{E}\right) \neq 1$. In fact, as we said in the introduction, this can be done in fact in a Borel way, as we now show. We will need the following simple fact.

Lemma 3.3. Let $\mu$ be a measure on $X, A$ and $B$ two Borel subsets of $X$, and set $K_{\mu}=\left\{\sigma \in S P\left(X^{2}\right): p_{0}(\sigma) \leq \mu\right.$ and $\left.p_{1}(\sigma) \leq \mu\right\}$. Then on $K_{\mu}$, the map $\sigma \mapsto \sigma(A \times B)$ is continuous.

Proof. Given $\epsilon>0$, pick $F_{A}$ compact and $U_{A}$ open with $F_{A} \subset A \subset U_{A}$ and $\mu\left(U_{A}-F_{A}\right)<\epsilon$, and similarly $F_{B}$ and $U_{B}$ for $B$. Then $\sigma\left(F_{A} \times F_{B}\right)<\sigma(A \times B)<$ $\sigma\left(U_{A} \times U_{B}\right)$, and for $\sigma$ in $K_{\mu}, \sigma\left(U_{A} \times U_{B}-F_{A} \times F_{B}\right)<\epsilon$. As $\sigma \mapsto \sigma\left(F_{A} \times F_{B}\right)$ and $\sigma \mapsto \sigma\left(U_{A} \times U_{B}\right)$ are respectively usc and lsc, we are done. 
Now let $E$ be a $K_{\sigma}$ equivalence relation, and let $E_{n}$ be an increasing sequence of compact symmetric sets in $X^{2}$, containing the diagonal, with $E=\bigcup_{n} E_{n}$. Fix $\mu$ in $P\left(X^{2}\right)$, and set $L_{n}=\left\{\sigma \in K_{\mu}: \sigma\left(X^{2}-E_{n}\right)=0\right\}$. Each $L_{n}$ is compact in $S P\left(X^{2}\right)$. Pick a sequence $\left(\sigma_{m}^{n}\right)_{m \in \mathbb{N}}$ of nonzero measures which is dense in $L_{n}$, with $\sigma_{0}^{0}=\mu^{\prime}$ (the image of $\mu$ on the diagonal of $X^{2}$ ). Finally set

$$
\sigma=\sum_{n} 2^{-\phi(m, n)-2} \cdot \frac{1}{\sigma_{m}^{n}(1)}\left(\sigma_{m}^{n}+\bar{\sigma}_{m}^{n}\right),
$$

where $\phi$ is a bijection between $\mathbb{N}^{2}$ and $\mathbb{N}$, and $\bar{\sigma}_{m}^{n}$ is symmetric to $\sigma_{m}^{n}$. Clearly $\sigma$ is a symmetric probability measure on $X^{2}$ which is supported by $E$, and its two projections are measure-equivalent to $\mu$. So $\sigma$ is adapted to $(E, \mu)$.

Lemma 3.4. $\mathcal{B}_{\mu}(\sigma)=\mathcal{B}_{\mu}(E)$.

Proof. Suppose not. Then the proof of Theorem 1.1 means that there is a Borel set $B$ with $\sigma\left(B^{2} \cup(X-B)^{2}\right)=1$ and such that $c(B)>\mu(B)$, where if we set

$$
H=\left\{\theta \in S P\left(X^{2}\right): \theta\left(X^{2}-E\right)=0 \text { and } p_{0}(\theta) \leq\left.\mu\right|_{B}\right\}
$$

and

$$
H_{n}=\left\{\theta \in S P\left(X^{2}\right): \theta\left(X^{2}-E_{n}\right)=0 \text { and } p_{0}(\theta) \leq\left.\mu\right|_{B}\right\}
$$

we have

$$
\begin{aligned}
c(B) & =\sup \{\mu(C): C \text { Borel } E \text {-invariant, } \mu(B-C)=0\} \\
& =\sup \left\{\mu \wedge \nu(X): \exists \theta \in H\left(\nu \ll p_{1}(\theta)\right)\right\} \\
& =\sup _{k} \sup _{n}\left\{\mu \wedge \nu(X): \exists \theta \in H_{n}\left(\nu \leq k p_{1}(\theta)\right)\right\} .
\end{aligned}
$$

Hence there exist $k, n \in \mathbb{N}$ and $\theta \in H_{n}$ such that $\mu \wedge k \cdot p_{1}(\theta)(X)>\mu(B)$. Let $f \leq k$ be such that $\mu \wedge k \cdot p_{1}(\theta)=p_{1}(f \theta)$. Then $\frac{1}{k} f \theta$ is in $L_{n}$. Moreover one must have $f \theta(B \times X-B)>0$, for otherwise $f \theta$ would be supported by $B^{2}$, and one would have $p_{1}(f \theta)(X)=p_{1}(f \theta)(B) \leq \mu(B)$. By Lemma 3.3, there is by continuity some $m \in \mathbb{N}$ such that $\sigma_{m}^{n}(B \times X-B)>0$; hence a fortiori $\sigma(B \times X-B)>0$, a contradiction which finishes the proof.

Theorem 3.5. Let $E$ be a $K_{\sigma}$ equivalence relation on $X$.

(i) There is a Borel (1st class) function $\varphi: P(X) \rightarrow P\left(X^{2}\right)$ such that for any $\mu \in P(X), \varphi(\mu)$ is adapted to $(E, \mu)$ and $\mathcal{B}_{\mu}(\varphi(\mu))=\mathcal{B}_{\mu}(E)$.

(ii) There are Borel functions $\psi_{n}: P(X) \times X \rightarrow \mathbb{R}$ such that for all $\mu \in P(X)$, the sequence $\psi_{n}(\mu,$.$) is norm-dense in \mathcal{B}_{\mu}(E)$.

(iii) There is a Borel function $\theta: P(X)-\mathcal{E}(E) \rightarrow \mathcal{K}(X)$ such that for non-Eergodic $\mu, 0<\mu\left([\theta(\mu)]_{E}\right)<1$.

Proof. Parts (ii) and (iii) follow easily from part (i), using again the Hopf ergodic theorem: For symmetric $\sigma \in P\left(X^{2}\right)$, and a sequence $\left(f_{m}\right)$ norm-dense in $\mathcal{C}(X)$, the functions $g_{m}^{\sigma}=\lim _{N} \frac{1}{N} \sum_{i=0}^{N-1} T_{\sigma}^{i} f_{m}$ form a sequence which is norm-dense in $\mathcal{B}_{\mu}(\sigma)$ by this theorem, and it is easy to get, in a Borel way in $\sigma$, representatives of this sequence. Composing with the function $\varphi$ given by (i) gives (ii), and (iii) follows easily. To get (i), it is enough by Lemma 3.4 to check that one can find for each $n$ in a Borel (and in fact 1st class) way in $\mu$, a sequence $\left(\sigma_{m}^{n}(\mu)\right)_{m \in \mathbb{N}}$ dense in $L_{n}(\mu)=\left\{\theta \in K_{\mu}: \theta\left(X^{2}-E_{n}\right)=0\right\}$. And this is easy, as $\left\{(\mu, \theta): \theta \in L_{n}(\mu)\right\}$ is compact in $P(X) \times P\left(X^{2}\right)$. 
Remark. The previous result is clearly uniform in $E$, via some natural encoding of the $K_{\sigma}$ equivalence relations on $X$ : We can e.g. encode $E$ by a sequence $\left(K_{n}\right) \in$ $\mathcal{K}\left(X^{2}\right)^{\mathbb{N}}$ which generates it, and the maps $\varphi^{E}, \psi_{n}^{E}$ and $\theta^{E}$ given by 3.5 can easily be taken Borel in the sequence $\left(K_{n}\right)$ too. It follows that the Borel class of $\mathcal{E}(E)$, for $K_{\sigma} E$, is bounded independently of $E$. In fact, $(E, \mu) \mapsto \varphi^{E}(\mu)$ can be chosen first class, and for each $m,(\sigma, x) \mapsto T_{\sigma}^{n} f_{m}(x)$ can be chosen of finite Borel class (depending on $n$ ), so that one can show that for a $K_{\sigma}$ equivalence relation $E, \mathcal{E}(E)$ is $\boldsymbol{\Pi}_{\omega+2}^{0}$. We do not know how sharp this computation is.

\section{REFERENCES}

[D] A. Ditzen, Definable equivalence relations on Polish spaces, Ph.D. Thesis, Caltech (1992).

[Ke] A.S. Kechris, Lectures on definable group actions and equivalence relations, forthcoming monograph.

[KL] A.S Kechris, A. Louveau, Descriptive Set Theory and the Structure of Sets of Uniqueness, London Mat. Soc. Lecture Notes Series 128, Cambridge Univ. Press, 1987. MR 90a:42008

[Kr] U. Krengel, Ergodic theorems, de Gruyter Berlin-New York (1985). MR 87i:28001

Equipe D'Analyse, Université Paris Vi, 75230 Paris Cedex 5, France

E-mail address: louveau@ccr.jussieu.fr

E-mail address: gam@jussieu.fr 